\title{
A Foreign Body in the Appendix
}

\author{
Satoshi Ogawa*, Yoshito Uenoyama, and Yukitaka Yamashita \\ Department of Gastroenterology and Hepatology, Japanese Red Cross Society Wakayama Medical Center, Wakayama, Japan
}

\section{Clinical image}

A 77-year-old man was referred to our hospital following ingestion of a dental drill bit. He was asymptomatic and had no abnormal findings on physical assessment or blood tests. We performed esophagogastroduodenoscopy but could not locate the drill bit. The next day, an abdominal radiograph showed the drill bit in the right lower abdominal quadrant. Two days later, we performed colonoscopy because the drill bit had not moved on the radiograph. However, we could not locate the drill bit. Subsequent CT examination revealed the drill bit within the appendix (Figure 1). Colonoscopy under fluoroscopic guidance showed the drill bit in the appendix, which was crookedly oriented (Figure 2). We unsuccessfully attempted to straighten the appendix with an endoscopic retrograde cholangiopancreatography catheter and endoscopically remove the drill bit. Although the patient was asymptomatic, laparoscopic appendectomy was performed. Pathological examination showed a $35-\mathrm{mm}$ drill bit within the appendix (Figure 3), and histological examination showed only

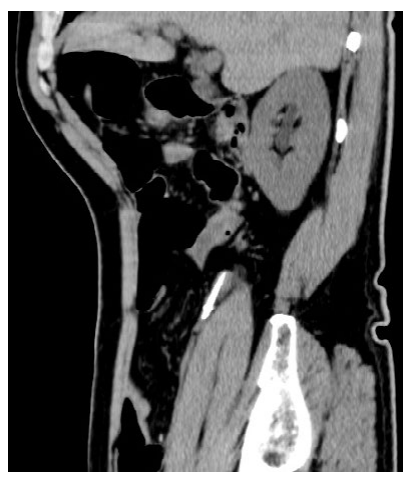

Figure 1. CT (sagittal view) showed the drill bit in the appendix.

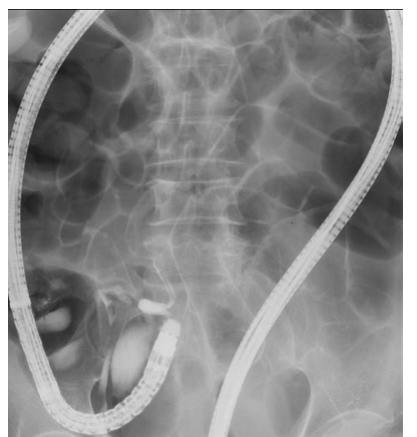

Figure 2. Colonoscopy under fluoroscopic guidance showed the drill bit in the appendix.

Copyright: (2016 Ogawa S. This is an open-access article distributed under the terms of the Creative Commons Attribution License, which permits unrestricted use, distribution, and reproduction in any medium, provided the original author and source are credited.

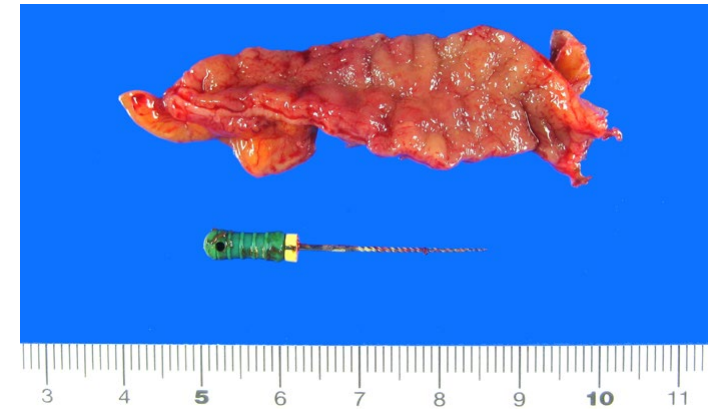

Figure 3. Pathological examination showed a $35-\mathrm{mm}$ drill bit within the appendix.

mild inflammation. The patient was discharged with no postoperative complications.

Eighty percent of ingested objects that reach the stomach are passed spontaneously without complications [1]. However, if the anatomical position of the object does not change on radiographs, especially when the object is present in the right lower abdominal quadrant, colonoscopy should be attempted to remove the object [2].

Balch et al reported that foreign bodies in the appendix comprised pins in $37 \%$ of cases, bullets in $22 \%$, and bone in $8 \%$. Sharp foreign bodies in the appendix cause perforations in $70 \%$. If endoscopic removal fails, prophylactic appendectomy is recommended [3].

\section{Authorship and contributorship}

Dr. Ogawa drafted the article. Dr. Uenoyama and Dr. Yamashita contributed the critical revision of the article for important intellectual content, and contributed the final approval of the article.

\section{Conflict of interest}

All authors declare no conflict of interests for this article.

\section{References}

1. Selivanov V, Sheldon GF, Cello JP, Crass RA(1984) Management of foreign body ingestion. Ann Surg 199: 187-191. [Crossref]

2. Klingler PJ, Seeling MH, DeVault KR, Wetscher GJ, Floch NR (1998)Ingested foreign bodies within the appendix: A 100-year review of the literature. Dig Dis 16: 308-314. [Crossref]

3. Balch CM, Silver D (1971)Foreign bodies in the appendix.Report of eight cases and review of the literature. Arch Surg 102: 14-20. [Crossref]

Correspondence to: Satoshi Ogawa, Department of Gastroenterology and Hepatology, Japanese Red Cross Society Wakayama Medical Center, 4-20 Komatsubaradori, Wakayama 640-8558, Japan; Tel: +81-73-4224171; Fax: +81 73-4261168; E-mail: aragorn_sato@yahoo.co.jp

Received: June 17, 2016; Accepted: July 02, 2016; Published: July 05, 2016 